\title{
Effect of strong magnetic field on surface polaritons in $\mathrm{ZnO}$
}

\author{
E.F. Venger ${ }^{1}$, A.I. Ievtushenko², L.Yu. Melnichuk ${ }^{2}$, O.V. Melnichuk ${ }^{2}$ \\ ${ }^{I} V$. Lashkaryov Institute of Semiconductor Physics, NAS of Ukraine \\ 41, prospect Nauky, 03028 Kyiv, Ukraine \\ ${ }^{2}$ Mykola Gogol Nezhyn State University \\ 2, Kropyv'yanskogo str., 16600 Nezhyn, Ukraine; e-mail: mov310@mail.ru
}

\begin{abstract}
Using the attenuated total reflectance technique, we studied the effect of strong uniform magnetic field $\vec{H}$ on the main properties of surface polaritons in $\mathrm{ZnO}$ single crystals. The used orientations were $C\|y, \vec{k} \perp C, x y\| C, \vec{H} \perp \vec{k}, \vec{H} \| y, k_{x}=k$, $k_{y, z}=0$; the free charge carrier concentration varied from $9.3 \times 10^{16}$ up to $2.0 \times 10^{18} \mathrm{~cm}^{-3}$. It has been shown that three dispersion curves exist in the zinc oxide single crystals for the above orientation. The possibility of excitation of an additional dispersion branch in optically anisotropic semiconductors placed into magnetic field is found using $\mathrm{ZnO}$ single crystals as an example. The damping coefficients for surface phonon and plasmon-phonon polaritons have been determined.
\end{abstract}

Keywords: $\mathrm{ZnO}$ single crystal, strong magnetic field, surface polariton, damping coefficient.

Manuscript received 16.09.09; accepted for publication 08.07.10; published online 30.09.10.

\section{Introduction}

Zinc oxide belongs to II-VI semiconductor compounds and crystallizes in the wurtzite structure. Its unique physico-chemical properties are required for application in metallurgy, space engineering, acousto-, micro- and optoelectronics as well as other areas of science, engineering and medicine [1]. In particular, continuous growth of application of $\mathrm{ZnO}$ single crystals in electronic-optical engineering takes place. This promotes both theoretical and experimental investigations of electrical, magnetic and optical properties of zinc oxide.

In [2], the spectra of external reflection of light from surface of $\mathrm{ZnO}$ (with the electron concentration from $10^{16}$ up to $\left.5 \times 10^{19} \mathrm{~cm}^{-3}\right)$ were studied experimentally and interconsistent parameters of $\mathrm{ZnO}$ single crystal bulk were obtained. It was shown that $\mathrm{ZnO}$ single crystals are characterized by strong anisotropy of properties of in the phonon subsystem, while those of the plasma subsystem demonstrate insignificant anisotropy. The authors of [3] used their experimental data to perform theoretical investigation of the coefficient of external reflection from polar uniaxial optically anisotropic single crystals of zinc oxide placed into strong uniform magnetic field. They found, for the first time, that there are some regions in the spectra of external reflection from the above single crystals where new oscillations appear. These oscillations are caused by the action of strong uniform magnetic field. The interaction between phonons and plasmons in $\mathrm{ZnO}$ single crystals placed into strong uniform magnetic field was studied. Similar results were obtained also for $\mathrm{SiC}$ single crystals (polytype $6 \mathrm{H}$ ) that are characterized by insignificant anisotropy of properties of in the phonon subsystem, while their plasma subsystem demonstrates strong anisotropy of its properties.

In $[5,6]$, a possibility of excitation of various phonon and plasmon-phonon polaritons in polar optically anisotropic $\mathrm{ZnO}$ semiconductor was studied. It was shown in [6] that one can excite the first- and second-type surface polaritons (SP) in the region of residual rays. However, we did not find in the literature information on investigations of properties of optically anisotropic semiconductors (placed into strong magnetic field) performed using the attenuated total reflectance (ATR) technique, with allowance made for the plasmonphonon interaction.

In this work, we studied the effect of strong uniform magnetic field on the properties of surface phonon and plasmon-phonon polaritons in optically anisotropic semiconductor (with $\mathrm{ZnO}$ single crystal serving as an example) for the orientations $C \| y$, $\vec{k} \perp C, x y\|C, \vec{H} \perp \vec{k}, \vec{H}\| y, k_{x}=k, k_{y, z}=0$ ( $C$ is the optical axis of the crystal). 


\section{Experimental procedure}

The ATR spectra of zinc oxide were measured using a spectrometer ИКС-29M with an attachment НПВО-2, according to the procedure considered in [2]. A semicylinder KRS-5 (index of refraction $n_{0}=2.38$ ) served as ATR element. An air gap (width $d=2 \div 15 \mu \mathrm{m}$ ) was made between the ATR prism and $\mathrm{ZnO}$ single crystal to be studied. The accuracy of angle adjustment in the attachment HПBO-2 with an element KRS-5 corresponded to $0.1^{\circ}$. The spectral width of the slit in the course of ATR spectra recording with a polarizer was $3 \mathrm{~cm}^{-1}$; the polarization coefficient was 0.98 . The accuracy of SP frequency determination was $\pm 1 \mathrm{~cm}^{-1}$. The sample sizes were chosen in such a manner that one could obtain the ATR spectra in the $p$-polarized light at the above-mentioned orientations of the wave vector $\vec{k}$, crystal optical axis $C$ and magnetic field $\vec{H}$.

\section{Theory}

Let us consider the polar optically anisotropic $\mathrm{ZnO}$ single crystal in which excitation and propagation of various SP occur along the semiconductor surface (see Fig. 1). The $x$-axis lies along the direction of electromagnetic wave propagation, and $C \| y$ ( $p$ polarization).

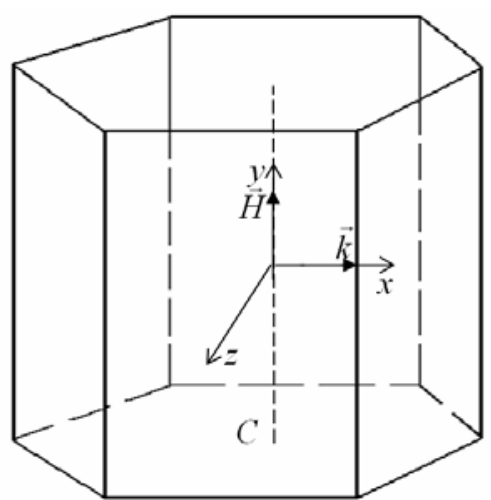

Fig. 1. Relative position of the vectors $\vec{H}, \vec{k}$ and coordinate axes $x, y, z$ in $\mathrm{ZnO}$ single crystals.
The ATR coefficient $I / I_{0}$ in the IR spectral region (here $I$ and $I_{0}$ are the intensities of IR radiation of a given frequency with and without a sample, respectively) was calculated with the expressions given in [2]. The contributions from the phonon and plasma subsystems of the semiconductor were taken to be additive, and allowance was made for the effect of strong uniform magnetic field on the single crystal. The agreement between the theoretical results and experimental data was achieved by usage in calculations of the components of permittivity tensor in magnetic field:

$$
\begin{aligned}
& \varepsilon_{1}=\varepsilon_{\infty \perp, \|}\left(1+\frac{\mathrm{v}_{L \perp, \|}^{2}-\mathrm{v}_{T \perp, \|}^{2}}{\mathrm{v}_{T \perp, \|}^{2}-\mathrm{v}^{2}-i \mathrm{Y}_{f \perp, \|} \mathrm{v}}+\frac{\mathrm{v}_{p \perp, \|}^{2}\left(\mathrm{v}+i \gamma_{p \perp, \|}\right)}{\mathrm{v}\left(\Omega^{2}-\left(\mathrm{v}+i \gamma_{p \perp, \|}\right)^{2}\right)}\right), \\
& \varepsilon_{2}=\frac{\varepsilon_{\infty \perp,\|,\|} \mathrm{v}_{p \perp, \|}^{2} \Omega}{\mathrm{v}\left(\left(\mathrm{v}+i \gamma_{p \perp, \|}\right)^{2}-\Omega^{2}\right)^{2}} \\
& \varepsilon_{3}=\varepsilon_{\infty \perp, \|}\left(1+\frac{\mathrm{v}_{L \perp, \|}^{2}-\mathrm{v}_{T \perp, \|}^{2}}{\mathrm{v}_{T \perp, \|}^{2}-\mathrm{v}^{2}-i \bigvee_{f \perp, \|} \mathrm{v}}+\frac{\mathrm{v}_{p \perp, \|}^{2}}{\mathrm{v}\left(\mathrm{v}+i \gamma_{p \perp, \|}\right)}\right),
\end{aligned}
$$

where $\Omega=\frac{e H}{m c}$ is the cyclotron frequency, $\varepsilon_{\infty \perp, \|}-$ high-frequency permittivity, $v_{L \perp, \|}, v_{T \perp, \|}$ - frequencies of the longitudinal and transverse optical phonons, respectively, $v_{p \perp, \|}-$ plasma resonance frequency, $\gamma_{p \perp, \|}$ - plasmon damping frequency, and $\gamma_{f \perp, \|}$ - optical phonon damping frequency.

Tables 1 and 2 present the interconsistent bulk and electrophysical parameters of the optically anisotropic $\mathrm{ZnO}$ single crystals. The parameters were obtained with the analysis of variance of the IR reflectance spectra in the region of plasmon-phonon interaction and using polarized light at different angles of incidence [2].

Table 1. Bulk parameters of $\mathrm{ZnO}$ single crystals grown using the hydrothermal method.

\begin{tabular}{|c|c|c|c|c|}
\hline $\mathrm{ZnO}$ & $\varepsilon_{0}$ & $\varepsilon_{\infty}$ & $v_{T}, \mathrm{~cm}^{-1}$ & $v_{L}, \mathrm{~cm}^{-1}$ \\
\hline$E \perp C$ & 8.1 & 3.95 & 412 & 591 \\
\hline$E \| C$ & 9.0 & 4.05 & 380 & 570 \\
\hline
\end{tabular}

Table 2. Electrophysical parameters of $\mathrm{ZnO}$ single crystals.

\begin{tabular}{|c|c|c|c|c|c|c|c|c|c|}
\hline \multirow{2}{*}{ Sample } & \multirow{2}{*}{$n, \mathrm{~cm}^{-3}$} & \multicolumn{2}{|c|}{$v_{p}, \mathrm{~cm}^{-1}$} & \multicolumn{2}{|c|}{$\gamma_{p}, \mathrm{~cm}^{-1}$} & \multicolumn{2}{|c|}{$\gamma_{f}, \mathrm{~cm}^{-1}$} & \multirow{2}{*}{$m_{\|}$} & \multirow{2}{*}{$m_{\perp}$} \\
\cline { 3 - 10 } & & $E \perp C$ & $E \| C$ & $E \perp C$ & $E \| C$ & $E \perp C$ & $E \| C$ & & \\
\hline ZO2-3 & $9.3 \times 10^{16}$ & 90 & 100 & 150 & 170 & 11 & 11 & 0.21 & 0.258 \\
\hline ZO1-3 & $6.6 \times 10^{17}$ & 240 & 250 & 280 & 260 & 13 & 13 & 0.23 & 0.260 \\
\hline ZO6-B & $2.0 \times 10^{18}$ & 420 & 480 & 406 & 350 & 21 & 21 & 0.22 & 0.260 \\
\hline
\end{tabular}




\section{Results and discussion}

Shown in Fig. 2 (curves 1,2, and 3) are the calculated (curve) ATR spectra $I / I_{0}$ for undoped zinc oxide $\left(n=9.3 \times 10^{16} \mathrm{~cm}^{-3}\right)$ taken at the air gap $d=14.6\left(1,1^{\prime}\right)$, $8.7\left(2,2^{\prime}\right)$ and $4.1 \mu \mathrm{m}\left(3,3^{\prime}\right)$, and angles of $30^{\circ}\left(1,1^{\prime}\right)$, $35^{\circ}\left(2,2^{\prime}\right)$ and $50^{\circ}\left(3,3^{\prime}\right)$. The calculations were performed with the expressions from [2] for the case of absent magnetic field. The dots show the experimental data for the sample $\mathrm{ZO} 2-3$ that were measured with the spectrometer ИКС-29M using the procedure considered in [2]. The width of the air gap between the ATR prism and sample $\mathrm{ZO} 2-3$ was varied until the intensity of the absorbed wave did not exceed $20 \%$, while the frequency of the minimum in the ATR spectrum remained constant [7]. The curves $1^{\prime}, 2^{\prime}, 3^{\prime}$ show the calculated ATR spectra for a $\mathrm{ZnO}$ single crystal placed into uniform magnetic field. The calculations for the sample $\mathrm{ZO} 2-3$ were made for the case when magnetic field reached $100 \mathrm{kOe}$.

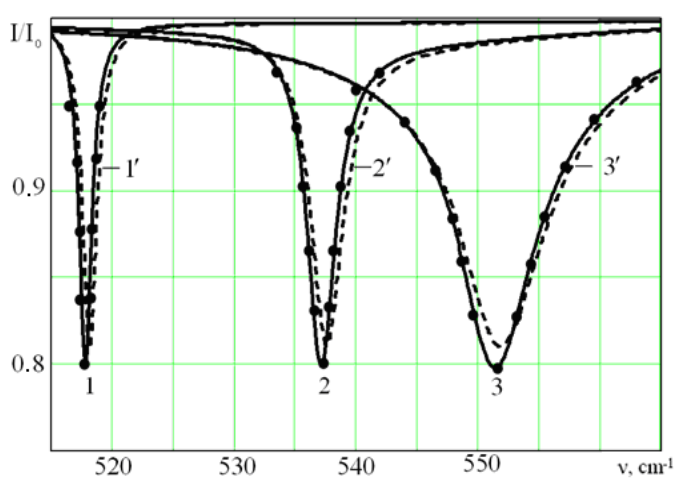

Fig. 2. The ATR spectra of $\mathrm{ZnO}$ (sample $\mathrm{ZO} 2-3$ ): 1-3$H=0 ; 1^{\prime}-3^{\prime}-H=100 \mathrm{kOe} ; 1,1^{\prime}-d=14.6 \mu \mathrm{m}, \varphi=30^{\circ} ; 2,2^{\prime}$ $-d=8.7 \mu \mathrm{m}, \varphi=35^{\circ} ; 3,3^{\prime}-d=4.1 \mu \mathrm{m}, \varphi=50^{\circ}$. Full and broken curves - calculation; dots - experiment.

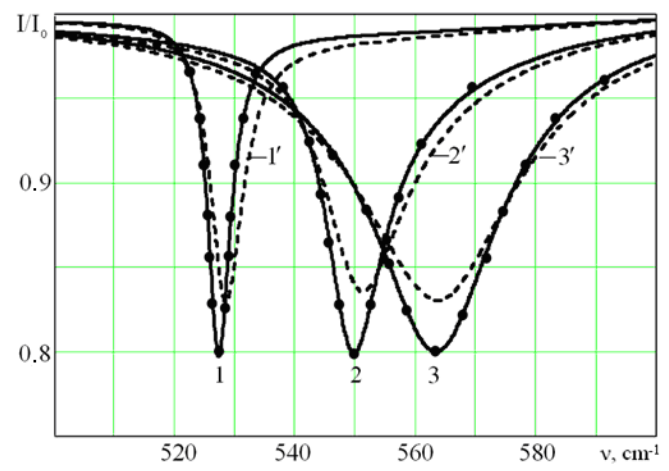

Fig. 3. The ATR spectra of $\mathrm{ZnO}$ (sample $\mathrm{ZO1}-3$ ): 1-3$H=0 ; \quad 1^{\prime}-3^{\prime}-H=100 \mathrm{kOe} ; 1,1^{\prime}-d=13 \mu \mathrm{m} ; 2,2^{\prime}-$ $d=7.4 \mu \mathrm{m} ; 3,3^{\prime}-d=3.4 \mu \mathrm{m}$. Full and broken curves calculation; dots - experiment.
The minima of the experimental and calculated spectra correspond to the SP frequencies $v_{\min }=518(1)$, 537 (2) and $551 \mathrm{~cm}^{-1}$ (3) (without magnetic field) and $v_{\min }=518\left(1^{\prime}\right), \quad 538\left(2^{\prime}\right)$ and $552 \mathrm{~cm}^{-1}\left(3^{\prime}\right)$ (with magnetic field). The half-widths of the spectra are, correspondingly, $\Gamma_{s}=2,3$ and $9 \mathrm{~cm}^{-1}$.

Fig. 3 presents the ATR spectra (taken in the absence of magnetic field) for a low-doped zinc oxide single crystal $\left(n=6.6 \times 10^{17} \mathrm{~cm}^{-3}\right)$, the angles of light incidence onto the ATR prism being $30^{\circ}\left(1,1^{\prime}\right)$, $35^{\circ}\left(2,2^{\prime}\right)$ and $50^{\circ}\left(3,3^{\prime}\right)$. The width of the air gap between the sample $\mathrm{ZO1}-3$ and ATR prism varied from $13(1)$ down to $3.4 \mu \mathrm{m}(3)$. The minima of the spectra correspond to the SP frequencies $v_{\min }=527(1)$, 550 (2), and $563 \mathrm{~cm}^{-1}$ (3), while the half-widths of the spectra are $\Gamma_{s}=5(1), 17$ (2) and $28 \mathrm{~cm}^{-1}$ (3). The curves $1^{\prime}, 2^{\prime}, 3^{\prime}$ are calculated with allowance for the effect of uniform magnetic field $(100 \mathrm{kOe})$ on the zinc oxide single crystal (sample ZO1-3). The frequencies of minima and half-widths of the ATR spectra are, correspondingly, $\quad v_{\min }=528\left(1^{\prime}\right), \quad 551\left(2^{\prime}\right), \quad$ and $564 \mathrm{~cm}^{-1}\left(3^{\prime}\right), \Gamma_{s}=7\left(1^{\prime}\right), 19\left(2^{\prime}\right)$, and $32 \mathrm{~cm}^{-1}\left(3^{\prime}\right)$. The dots show the experimental results for the $\mathrm{ZnO}$ single crystal (sample $\mathrm{ZO1}-3$ ).

Shown in Fig. 4 (curves 1, 2, and 3) are the calculated ATR spectra for heavily doped $\mathrm{ZnO}$ $\left(n=2.0 \times 10^{18} \mathrm{~cm}^{-3}\right)$. The angles of incidence were $30^{\circ}\left(1,1^{\prime}\right), 35^{\circ}\left(2,2^{\prime}\right)$ and $50^{\circ}\left(3,3^{\prime}\right)$; the widths of the air gaps between the ATR prism and zinc oxide were, correspondingly, $\quad 10.2\left(1,1^{\prime}\right), \quad 5.6\left(2,2^{\prime}\right) \quad$ and $2.6 \mu \mathrm{m}\left(3,3^{\prime}\right)$. The curves $1-3$ correspond to the case when magnetic field is absent. The curves (dotted) $1^{\prime}, 2^{\prime}$, $3^{\prime}$ show the ATR spectra calculated with allowance made for the effect of strong magnetic field $(100 \mathrm{kOe})$ on the $\mathrm{ZnO}$ single crystal, the orientation being $\vec{H} \perp \vec{k}$, $\vec{H} \| y$. The spectral minima correspond to the SP frequencies $v_{\min }=557\left(1^{\prime}\right), 574\left(2^{\prime}\right)$, and $582 \mathrm{~cm}^{-1}\left(3^{\prime}\right)$. The half-widths of the spectra are, correspondingly, $\Gamma_{s}=$ $58\left(1^{\prime}\right), 92\left(2^{\prime}\right)$, and $99 \mathrm{~cm}^{-1}\left(3^{\prime}\right)$.

Fig. 5 shows the ATR spectra for undoped zinc oxide (sample $\mathrm{ZO} 2-3$ ). The concentration of free charge carriers was $n=9.3 \times 10^{16} \mathrm{~cm}^{-3}$; the angle of incidence of IR radiation onto the ATR prism was $50^{\circ}$. The air gap between the ATR prism and $\mathrm{ZnO}$ single crystal was $4 \mu \mathrm{m}$. The magnetic field strength was varied: it took the values $0(1), 30(2), 65(3)$, and $100 \mathrm{kOe}(4)$. The spectral minimum was at the frequency $v_{\min }=(551 \pm 1) \mathrm{cm}^{-1}$ for all the values of magnetic field strength; $\Gamma_{s}=9 \mathrm{~cm}^{-1}$ for the curves $1-3$ and $10 \mathrm{~cm}^{-1}$ for the curve 4. 


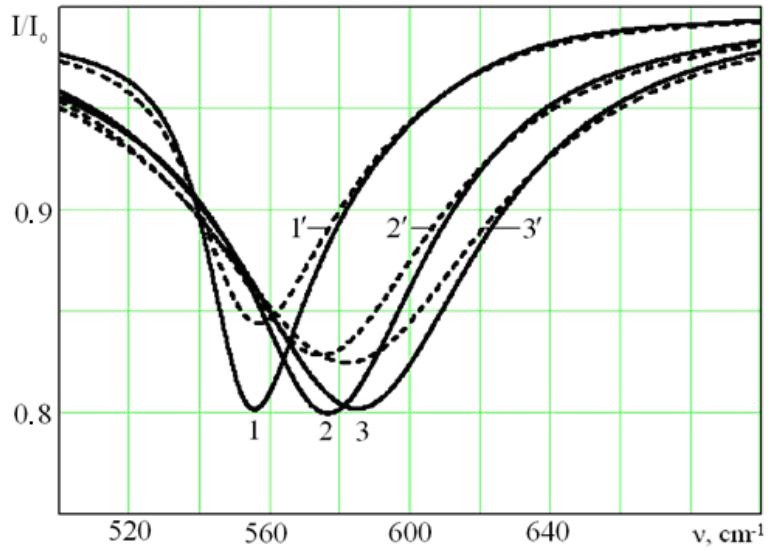

Fig. 4. The ATR spectra of $\mathrm{ZnO}$ (sample $\mathrm{ZO6}-\mathrm{B}$ ): 1-3$H=0 ; 1^{\prime}-3^{\prime}-H=100 \mathrm{kOe} ; 1,1^{\prime}-d=10.2 \mu \mathrm{m}, \varphi=30^{\circ} ; 2,2^{\prime}$ $-d=5.6 \mu \mathrm{m}, \varphi=35^{\circ} ; 3,3^{\prime}-d=2.6 \mu \mathrm{m}, \varphi=50^{\circ}$.

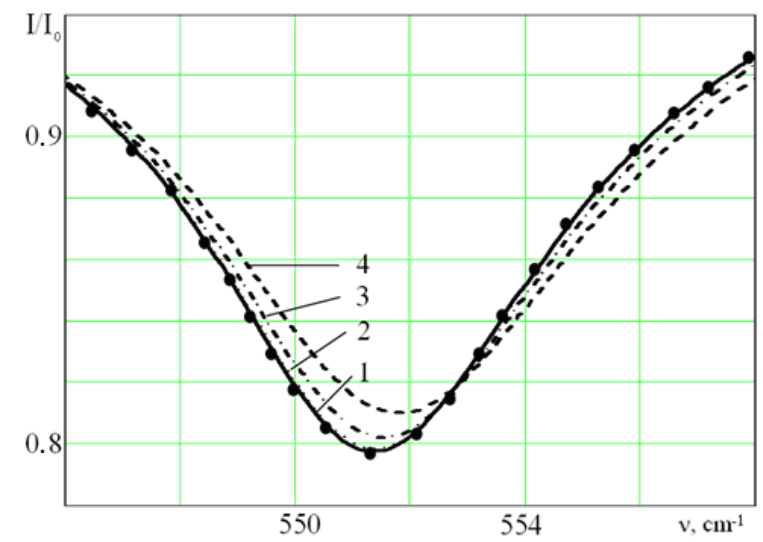

Fig. 5. The ATR spectra of $\mathrm{ZnO}$ (sample $\mathrm{ZO} 2-3$ ): $1-4-H=$ $0,30,65,100 \mathrm{kOe} ; d=4 \mu \mathrm{m}, \varphi=50^{\circ}$. Full and broken curves - calculation; dots - experiment.

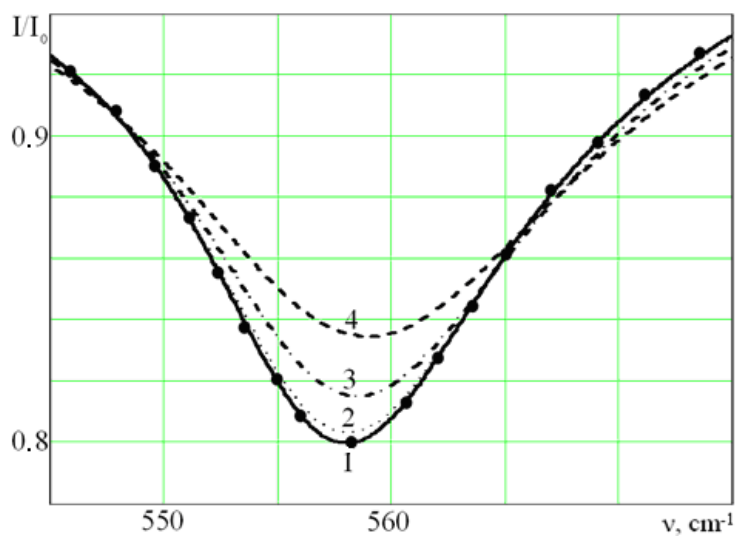

Fig. 6. The ATR spectra of $\mathrm{ZnO}$ (sample $\mathrm{ZO1}-3$ ): $1-4-H=$ $0,30,65,100 \mathrm{kOe} ; d=5 \mu \mathrm{m}, \varphi=40^{\circ}$. Full and broken curves - calculation; dots - experiment.
Fig. 6 demonstrates the effect of magnetic field on the ATR spectra. In our calculations, we used the data from Tables 1 and 2 for the low-doped sample ZO1-3, with the angle of incidence $40^{\circ}$ and air gap width $5 \mu \mathrm{m}$. The calculations were performed for magnetic fields of $0(1)$ and $30(2), 65(3)$ and $100 \mathrm{kOe}(4)$, respectively. From the ATR spectra, one can see that growth of magnetic field strength is accompanied with increase of both the ATR spectra half-width and SP damping. The position of the spectral minima remains the same: it corresponds to the SP frequency $v_{\min }=558 \mathrm{~cm}^{-1} . \Gamma_{s}$ is $20,22,25$ and $28 \mathrm{~cm}^{-1}$ for the curves $1-4$, respectively.

The experimental spectrum (Fig. 6, dots 1) was taken for the low-doped sample ZO1-3 at frequency scanning; both the angle of IR radiation incidence onto the ATR prism $\left(\varphi=40^{\circ}\right)$ and air gap width $(d=5 \mu \mathrm{m})$ remained constant. The increase of the concentration of free charge carriers up to $2.0 \times 10^{18} \mathrm{~cm}^{-3}$, at constant values $(30,65$ and $100 \mathrm{kOe})$ of the strength of uniform magnetic field, angle of incidence and air gap width $(4 \mu \mathrm{m})$, is accompanied with a shift of the SP frequency towards the low-frequency spectral region (see Fig. 7). The spectral minima correspond to the SP frequencies $v_{\min }=581(1), 580(2)$ and $578 \mathrm{~cm}^{-1}$ (3), respectively, and $\Gamma_{s}=83(1,2), 88(3)$ and $98 \mathrm{~cm}^{-1}(4)$. The curve 1 is calculated for the case of absent magnetic field.

One can see from Fig. 5 that magnetic field affects the absorption coefficient in the region of minimum of ATR spectrum and does not change the frequency of the minimum (within experimental errors). As the level of $\mathrm{ZnO}$ doping is being increased, the effect of magnetic field on the character of ATR spectrum becomes more substantial (see Figs 4 and 7).

Shown in Fig. 8 are the dispersion curves of zinc oxide single crystals when there is no magnetic field and allowance is made for the damping coefficients of the phonon and plasma subsystems. The concentration of free charge carriers (electrons) in $\mathrm{ZnO}$ varied from $n=9.3 \times 10^{16} \mathrm{~cm}^{-3}\left(l\right.$ and $\left.l^{\prime}\right)$ up to $n=2.0 \times 10^{18} \mathrm{~cm}^{-3}$ ( 3 and $3^{\prime}$ ). The curves 2 and $2^{\prime}$ were taken at $n=6.6 \times 10^{17} \mathrm{~cm}^{-3}$. The curves $1-3$ correspond to the high-frequency dispersion branches with the limit frequency values $v^{+}(k)=561(1), \quad 578(2)$ and $627 \mathrm{~cm}^{-1}$ (3). The curves $I^{\prime}-3^{\prime}$ are calculated lowfrequency dispersion branches with the limit frequency values $v^{-}(k)=59\left(1^{\prime}\right), \quad 152\left(2^{\prime}\right)$ and $246 \mathrm{~cm}^{-1}\left(3^{\prime}\right)$. The SP dispersion curves were calculated with the expressions from [2, 6-9] and data from Tables 1 and 2. The dots represent the experimental values obtained using the ATR technique. The limit frequency values $v^{ \pm}$obtained from the ATR spectra of the samples under investigation are in agreement with the results of [6]. 


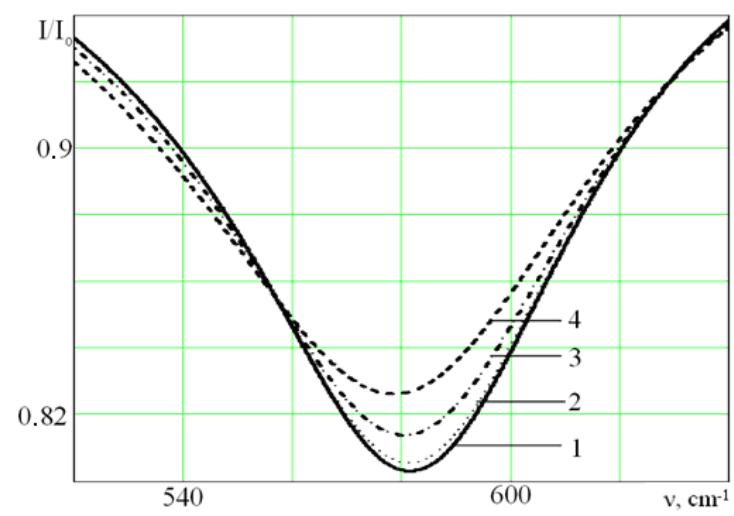

Fig. 7. The ATR spectra of $\mathrm{ZnO}$ (sample ZO6-B) ). 1-4$H=0,30,65,100 \mathrm{kOe} ; d=4 \mu \mathrm{m}, \varphi=40^{\circ}$.

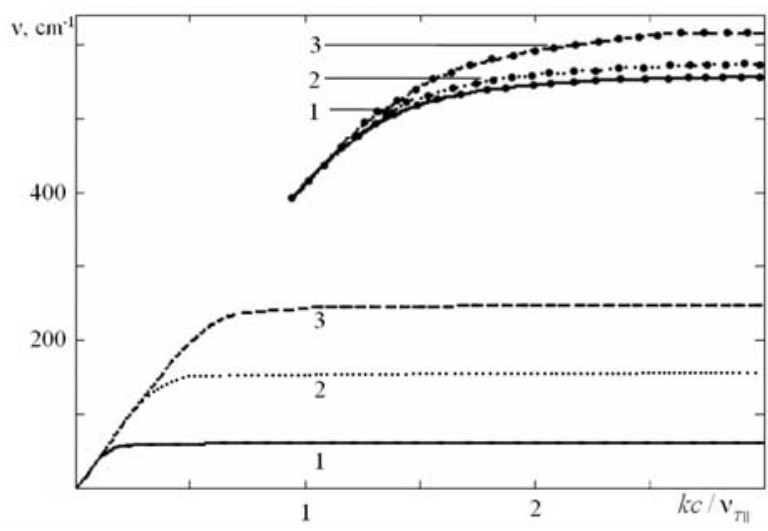

Fig. 8. The dispersion curves for $\mathrm{ZnO}$ single crystal. Curves calculation; dots - experiment.
One can see from Fig. 8 that increase in the concentration of free charge carriers (electrons) in $\mathrm{ZnO}$ is accompanied with a shift of the frequency of surface plasmon-phonon polaritons (SPPP) towards higher values. The SPPP high-frequency branches, whatever the degree of sample doping, begin from the frequency $v=v_{T \perp}$ and exist at $k>>2 \pi v / c$, approaching asymptotically the SP limiting frequency [2].

Fig. 9 presents the dispersion curves of low-doped $\mathrm{ZnO}$ single crystal (sample $\mathrm{ZO1}-3$ ) obtained under action of uniform magnetic field (from 0 up to $100 \mathrm{kOe}$ ); the orientation was stated above. One can see from Fig. 9 (inset) that the high-frequency dispersion curve remains practically the same as magnetic field varies from zero up to $100 \mathrm{kOe}$, while the lower dispersion branch shifts towards lower frequencies as magnetic field grows. Besides, as it follows from Fig. 8, action of magnetic field on the $\mathrm{ZnO}$ single crystal within the frequency range from 190 up to $350 \mathrm{~cm}^{-1}$ leads to appearance of one more dispersion branch (limited with the wave vector value) that shifts towards higher frequencies as the external magnetic field grows from $30 \mathrm{kOe}$. The reason is that, as the magnetic field strength increases, the cyclotron frequency $\Omega$ grows. In this case, coupling between plasmons and phonons breaks, and an additional dispersion branch appears [10]. The initial points for the lower and upper dispersion branches correspond to the frequencies $v=0$ and $v_{T}$ that are solutions of the equations $\varepsilon_{1}=1$ or $\varepsilon_{1}=0$. Asymptotes to the additional branch are specified by the equations $1+\varepsilon_{1} \pm i \varepsilon_{2}=0.3$ gives the limit frequencies of the additional dispersion branches for $\mathrm{ZnO}$ single crystals with allowance made for the above requirements.

Table 3. The limit frequency values for SP of different types in ZnO single crystals placed into magnetic field.

\begin{tabular}{|c|c|c|c|c|c|c|c|c|c|c|c|}
\hline$H$, Oe & \multicolumn{3}{|c|}{0} & \multicolumn{3}{|c|}{$3 \times 10^{4}$} & \multicolumn{3}{c|}{$6.5 \times 10^{4}$} & \multicolumn{3}{c|}{$10^{5}$} \\
\hline Sample & $v^{-}, \mathrm{cm}^{-1}$ & $v^{+}, \mathrm{cm}^{-1}$ & $v^{-}, \mathrm{cm}^{-1}$ & $v_{f}, \mathrm{~cm}^{-1}$ & $v^{+}, \mathrm{cm}^{-1}$ & $v^{-}, \mathrm{cm}^{-1}$ & $v_{f} \mathrm{~cm}^{-1}$ & $v^{+}, \mathrm{cm}^{-1}$ & $v^{-}, \mathrm{cm}^{-1}$ & $v_{f}, \mathrm{~cm}^{-1}$ & $\mathrm{v}^{+}, \mathrm{cm}^{-1}$ \\
\hline ZO2-3 & 59 & 561 & 34 & 108 & 561 & 21 & 171 & 561 & 14 & 242 & 561 \\
\hline ZO1-3 & 152 & 578 & 124 & 201 & 576 & 98 & 245 & 575 & 80 & 293 & 574 \\
\hline ZO6-B & 246 & 627 & 221 & 287 & 620 & 196 & 316 & 612 & 174 & 345 & 607 \\
\hline
\end{tabular}

Table 4. The half-width of the minimum $\Gamma_{s}$ in the ATR spectra and SP damping coefficient $\Gamma_{s p}$ in ZnO single crystals at $\gamma_{p}=0, \gamma_{f}=0 ; H=0$ and $100 \mathrm{kOe}$.

\begin{tabular}{|c|c|c|c|c|c|c|c|c|}
\hline \multirow{2}{*}{$\varphi$, deg. } & \multicolumn{4}{|c|}{$H=0$} & \multicolumn{4}{|c|}{$H=100 \mathrm{kOe}$} \\
\hline & $v_{\min }, \mathrm{cm}^{-1}$ & $\chi$ & $\Gamma_{s}, \mathrm{~cm}^{-1}$ & $\Gamma_{s p}, \mathrm{~cm}^{-1}$ & $v_{f}, \mathrm{~cm}^{-1}$ & $\chi$ & $\Gamma_{s}, \mathrm{~cm}^{-1}$ & $\Gamma_{s p}, \mathrm{~cm}^{-1}$ \\
\hline \multicolumn{9}{|c|}{ ZO1-3 } \\
\hline 30 & 527 & 1.647 & 23.42 & 17.74 & 528 & 1.645 & 39.43 & 24.83 \\
\hline 35 & 550 & 1.981 & 111.92 & 91.93 & 551 & 1.967 & 175.32 & 134.64 \\
\hline 50 & 564 & 2.499 & - & 626.82 & 564 & 2.408 & - & 618.52 \\
\hline \multicolumn{9}{|c|}{$\mathrm{ZO} 2-3$} \\
\hline 30 & 518 & 1.626 & 9.7 & 6.96 & 518 & 1.623 & 9.76 & 6.88 \\
\hline 35 & 537 & 1.936 & 34.16 & 28.08 & 538 & 1.927 & 43.07 & 34.96 \\
\hline 50 & 551 & 2.596 & 200.88 & 188.28 & 552 & 2.65 & 286.68 & 266.12 \\
\hline \multicolumn{9}{|c|}{ ZO6-B } \\
\hline 30 & 555 & 1.734 & 262.34 & 128.37 & 556 & 1.728 & 347.56 & 165.83 \\
\hline 35 & 576 & 1.96 & 556.89 & 345.16 & 574 & - & - & - \\
\hline 50 & 585 & - & - & - & 582 & - & - & - \\
\hline
\end{tabular}


The effect of magnetic field on the dispersion curves of undoped and heavily doped $\mathrm{ZnO}$ single crystals (samples $\mathrm{ZO} 2-3$ and $\mathrm{ZO} 6-\mathrm{B}$ ) is similar. Fig. 10 presents the calculated dispersion curves of $\mathrm{ZnO}$ placed into magnetic field of $100 \mathrm{kOe}$. One can see that, under action of magnetic field, all three dispersion branches shift towards higher frequencies as the concentration of free charge carriers grows.

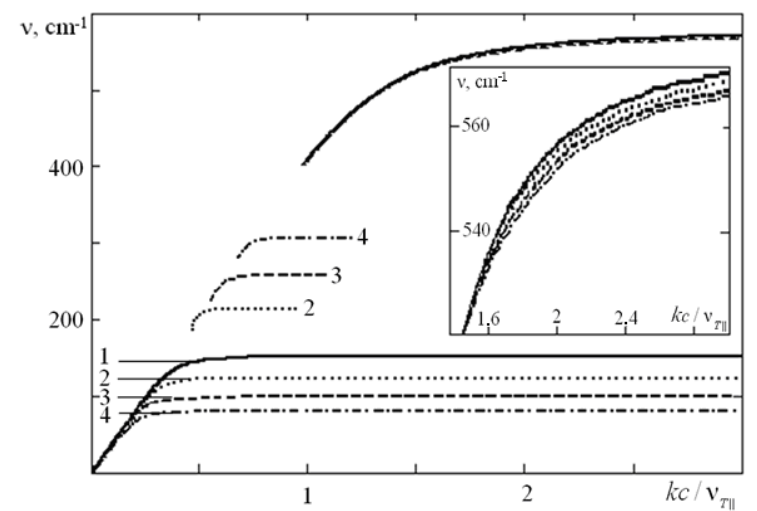

Fig. 9. The dispersion curves for $\mathrm{ZnO}$ single crystal (sample ZO1-3) with allowance made for the effect of magnetic field: $1-4-H=0,30,65,100 \mathrm{kOe}$.

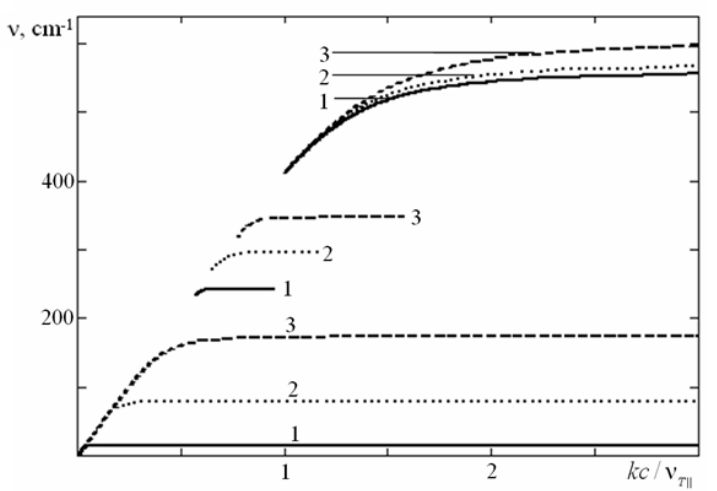

Fig. 10. Different types of dispersion curves for $\mathrm{ZnO}$ single crystals placed into magnetic field of $100 \mathrm{kOe}: 1-\mathrm{ZO} 2-3$; $2-\mathrm{ZO1}-3 ; 3-\mathrm{ZO} 6-\mathrm{B}$.

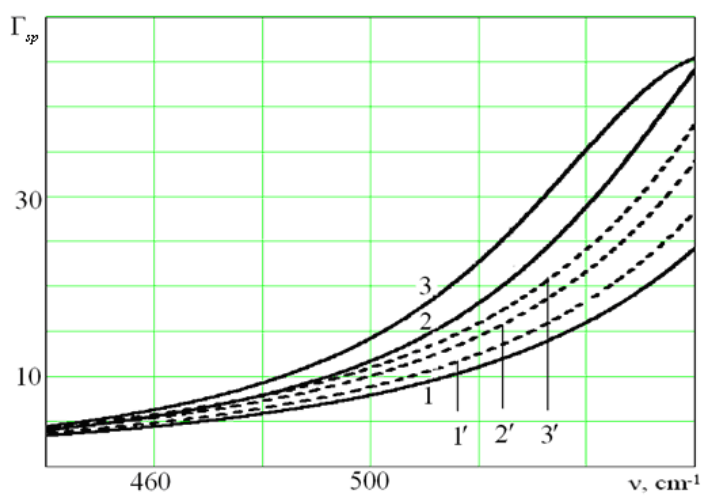

Fig. 11. The frequency dependence of SP damping coefficient, $\Gamma_{s p}(v)$, for $\mathrm{ZnO}$ single crystal: $1-\mathrm{ZO} 2-3 ; 2-\mathrm{ZO} 1-3 ; 3-$ ZO6 - B ; 1'-3' (sample ZO6 - B ) $H=30,65,100 \mathrm{kOe}$.

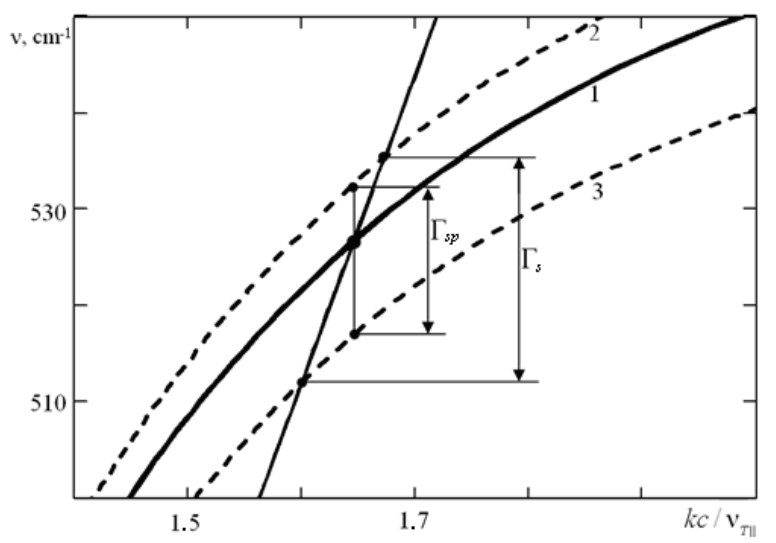

Fig. 12. The dispersion curve for the sample ZO1-3 [9]. $\Gamma_{p}$ and $\Gamma_{s p}$ - half-width and SP damping coefficient

Shown in Figs 9 and 10 are the dispersion curves calculated with allowance made for anisotropy of the phonon and plasma subsystems within the harmonic approximation. The investigations of dispersion curves (with allowance made for damping) were performed in [11] for magnetoplasmons and surface plasmon-phonon modes in magnetic field. The authors of [11] found that the dispersion curves in an optically isotropic medium calculated with consideration for damping of magnetoplasmons demonstrated bending backwards. As to the optically anisotropic crystals, no bending of dispersion curves was observed at the above orientation.

Fig. 11 shows the frequency dependences of the SP damping coefficient, $\Gamma_{s p}(v)$, for $\mathrm{ZnO}$ single crystal. The calculations were made for single crystals with ideally flat surface [2]. The curves 1-3 were calculated according to [2] for zinc oxide single crystals with different doping levels and without action of magnetic field. The curves $1^{\prime}-3^{\prime}$ show $\Gamma_{s p}(v)$ dependence for the heavily doped sample ZO6-B placed into magnetic field $\vec{H} \perp \vec{k}, \quad \vec{H} \| y \quad$ of $30\left(1^{\prime}\right), \quad 65\left(2^{\prime}\right) \quad$ and $100 \mathrm{kOe}\left(3^{\prime}\right)$.

The SP damping coefficient was determined with a graphical method in [2,9]. Fig. 12 shows the procedure used for determination of the SP damping coefficient $\Gamma_{s p}(v)$ for optically anisotropic $\mathrm{ZnO}$ single crystals [9]. The curve 1 is the dispersion branch, while the dashed curves 2 and 3 determine the half-width $\Gamma_{s}$ of the ATR spectrum. The "true" spectrum width, according to [2], is equal to the SP damping coefficient and is determined by the difference of the frequencies located at the intersection of the perpendicular to the abscissa axis in the system "dashed curves - dispersion point". Table 4 presents the calculated data $\Gamma_{s p}(v)$ and $\Gamma_{s}$ for $\mathrm{ZnO}$ single crystals with different doping levels under the conditions $\gamma_{p}=0, \gamma_{f}=0$, without and with magnetic field of $100 \mathrm{kOe}$. The angles of incidence onto the ATR prism were $30^{\circ}, 35^{\circ}$ and $50^{\circ}$.

One can see from Table 4 that, as the angle of incidence and concentration of free charge carriers in 
$\mathrm{ZnO}$ single crystal grow, the SP damping coefficient increases. The effect of plasmon-phonon damping and external magnetic field on $\Gamma_{s}$ and $\Gamma_{s p}$ in $\mathrm{ZnO}$ is similar.

\section{Conclusion}

Thus, action of strong uniform magnetic field on optically anisotropic $\mathrm{ZnO}$ single crystals (orientations $C\|y, \quad \vec{k} \perp C, \quad x y\| C, \quad \vec{H} \perp \vec{k}, \quad \vec{H} \| y, \quad k_{x}=k$, $\left.k_{y, z}=0\right)$ is accompanied by variation of the main properties of phonon and plasmon-phonon SP. For the first time, it has been shown that, in optically anisotropic $\mathrm{ZnO}$ single crystals subjected to action of uniform magnetic field, an additional dispersion branch may be excited. As the strength of the external magnetic field grows, that branch shifts towards higher frequencies. The initial frequency and character of the additional dispersion branch depend on the free charge carrier concentration and strength of magnetic field. The lower dispersion branch is deformed under action of magnetic field on the optically anisotropic single crystal, while the high-frequency branch of the dispersion curve remains the same (within experimental errors). As to the SP damping coefficient for $\mathrm{ZnO}$, this problem needs additional studies. However, from the analysis performed it follows that the effect of external magnetic field is unquestionable.

\section{References}

1. 1. S. Baruah, J. Dutta, Hydrothermal growth of $\mathrm{ZnO}$ nanostructures (Topical Review) // Sci. Technol. Adv. Mater. 10, p. 1-18 (2009).

2. E.F. Venger, O.V. Melnichuk, Yu.A. Pasichnyk, Residual Rays Spectroscopy. Nauka, Kyiv, 2001 (in Ukrainian).
3. E.F. Venger, A.I. Ievtushenko, L.Yu. Melnichuk, O.V. Melnichuk, Investigation of $\mathrm{ZnO}$ single crystals subjected to high uniform magnetic field in IR spectral range // Semiconductor Physics, Quantum Electronics \& Optoelectronics. 11(1), p. 6-10 (2008).

4. E.F. Venger, A.I. Ievtushenko, L.Yu. Melnichuk, O.V. Melnichuk, The reflection spectra of $6 \mathrm{H}-\mathrm{SiC}$ single crystal placed into strong uniform magnetic field // Inzhenerno-fizicheskii zhurnal, 82(6), p. 1187-1194 (2009), in Russian.

5. A.V. Melnichuk, Investigation of surface polaritons in zinc oxide single crystals // Functional Materials, 5(1), p. 25-30 (1998).

6. O.V. Melnichuk, L.Yu. Melnichuk, Yu.A. Pasechnik, Surface plasmon-phonon polaritons in hexagonal zinc oxide // Zhurnal Tekhnich. Fiziki, 68(1), p. 58-62 (1998), in Russian.

7. N.L. Dmitruk, V.G. Litovchenko, V.L. Strizhevskiy, Surface Polaritons in Semiconductors and Insulators. Naukova Dumka, Kiev, 1989 (in Russian).

8. O.V. Melnichuk, L.Yu. Melnichuk, Yu.A. Pasechnik, Effect of anisotropy on the dispersion curves of surface plasmon-phonon polaritons in zinc oxide // Fizika Tverdogo Tela 38(2), p. 651-653 (1996), in Russian.

9. Melnichuk A.V., Pasechnik Yu.A., Damping of surface plasmon-phonon polaritons in zinc oxide // Fiz. Tverd. Tela 38(8), p. 2343-2346 (1996) (in Russian).

10. Surface Polaritons: Electromagnetic Waves at Surfaces and Interfaces, Eds. V.M. Agranovich, D.L. Mills. North-Holland, Amsterdam, 1985.

11. O.V. Shramkova, Damping of electromagnetic waves in a semiconductor superlattice placed into magnetic field // Zhurnal Tekhnich. Fiziki, 74(2), p. $92-97$ (2004), in Russian. 